\title{
Note sur la théorie de surfaces réeiproques.
}

Par H. G. Zeuthen à Copenhague.

Dans mes deux mémoires au commencement de co volume des "Annalen" $j$ 'ai attribué aux surfaces dont je moccupe certaines siu. gularités que jai énumérées dans l'introduction du premier de ces némoires. J'ai supposé qu'aucune autre singulärité ne sty ajoutát, on bien que les sinģuarités se présentassent de la manière la plus genérale. Or, comme cette manière la plus générale est différente suivant qu'on regarde la surface comme lieu de points ou comme enveloppe de plans, il faut diviser les singularités en singularites de lifinition ponctuelle et en singularités de définition tangentiolle. Les singularites de définition ponctuelle sant celles qui satisferont de la manière la plus générale à leur détiaition si lón regarde la surface comme lieu de points, et les singularités de définition tangentielle sont celles qui y satisferont de la manière la plus générale si lon regarde la surface comme enveloppe de plans.

Nous n'avons pas dit expressément quelles défnitions de singum larités sont ponctuelles et quelles tangentielles, sans quoi les détinitions ne sont pas complètes. Nous supléerons ici i cette manque en montrant que la division que nous avons suipie, sans le dire, est la plus vaturelle. Comme une surface regardée comme lien de pointa n'a pas, en général, des courbes doubles et cuspidales, mais bien dess séries de plans tangents doubles et stationnaires, les définitions des singularités ordinaires des plans tangents doubles et stationnaires $\left(b^{\prime}, k, t^{\prime}, \gamma^{\prime}, q^{\prime}, o^{\prime}, c^{\prime}, h^{\prime}, \beta^{\prime}, r^{\prime}, \sigma^{\prime}\right)$ doivent etre ponctuelles, $t$ celles des singularités prdinaires de la conrbe double et de la courbe cuspidale $\left(b, k, t, \gamma, q, e, c, h_{\gamma} \beta, r, \sigma\right)$ doivent etre tangentielles. $\left.{ }^{*}\right)$ Wne

*) Alors on sait que les $\beta$ pointo cospidaux de la courbe cuspidale ue tronvent sur la courbe double, et que les $\gamma$ points cuapidax de la courbe double ve troupent sur la courbe euspidale, et ces point bont doue les raéme que ceux dont NM. Salmon et Cayley désignent les nombres de ia meme maniere. 1. Cayley m'a fait observer que les deux conrbes peurent avoir d'antres points cuspidaux. Ces nonveaux points singalier demanderajent une nouvelle étude. Jaurais dû dire expressepment que ma définition de $k$ (pombre v'lückerien de génératrices doubles d'un cône projetant la courbe doublè ent un peru différente de celle des MX. Salmon et Cayley, qua explique ane discordance apparente entre leurs formules et les miennes. J'eris $k-f-3 t-\Sigma(\cdots)$ or $\mathbf{u}$. Cayleg Ecrit sedepent $k$. 
courbe double, une fois attribuée à nne surface regardée comme lieu de points, sera en général douée de points-pinces, pendant que la courbe double d'une surface regardée comme enveloppe de plans n'en a pas généralement. Pour cette raison on doit domer à ces $j$ singrlarités une définition ponctuelle, et aux $j^{\prime}$ plans-pinces une définition tangentielle. De même les $\chi$ points-clos sont de définition ponctuelle, et les $\chi^{\prime}$ plans-clos de définition tangentielle. Nous avons donné expressément aux points coniques et aux singularités qui y appartiennent une définition ponctuelle, et aux plans qui sont tangents le long d'une courbe et aux singularités qui $\mathrm{y}$ appartiennent une définition tangentielle. C'est en regardant les définitions de $f$ et de $i$ comme ponctuelles, et celles de $f^{\prime}$ et de $i$ comme tangentielles, qu'on a les équations $f=f^{\prime}$ et $i=\ddot{t}$.

Nous faisons encore une autre distinction: les propriétés ponctuelles d'une singularité sont celles qui ont rapport aux points de la surface, et les propriétés tangentielles celles qui ont rapport à ses plans tangents. $t$ indique, par exemple, le nombre des plans qui ont la propriété ponctuelle de rencontrer la surface en une courbe douée de trois points doubles, et la propriété tangentielle d'être plans tangents triples, soit de la surface elle-même, soit de l'enveloppe des plans tangents doubles. Les propriétés ponctuelles des singularités de définition ponctuelle, ainsi que les propriétés tangentielles des singularités de définition tangentielle, sont indiquées dans les définitions elles-mêmes: mais il est souvent très-difficile de trouver les propriétés ponctuelles des singularités de définition tangentielle, et les propriétés tangentielles des singularités de définition ponetuelle, et ces propriétés peuvent être assez compliquées. Pour connaitre suffisamment les propriétés tangentielles d'un point-pince, par exemple, il faut avoir étudié la singularité de l'enveloppe des plans tangents stationnaires causée par le point-pince. Pour la trouver on a besoin d'étudier les singularités que lHessieune et sa courbe d'intersection avec la surface donnée out à ce même point.

Si lon regarde, dans une recherche, une surface comme lieu de ses points on doit conuaître les propriétés ponctuelles des singularités. qu'on lui attribue, et si l'on regarde nne surface comme enveloppe de ses plans tangents on doit connaitre les propriétés tangentielles de sess singularités. Daus une théorie des surfaces réciproques on a besoin de connaitre, à la fois, les propriétés ponctuelles et les propriétés tangentielles des singularités qu'on attribue aux surfaces. N'ayant fait une étude assez profonde ni des propriétés tangentielles des singularités $j, x, \eta$ et $\xi$, dont la définition est ponctuelle, ni des propriétés ponctuelles des singularités $j^{\prime}, \chi^{\prime}, \eta^{\prime}$ et $\xi$, dont la definition est tangentielle, je dois prier les lecteurs de mes mémoires dajouter wa 
mémoire "Sur deux surfaces dont les points se correspondent un-i-un", où les surfaces sont regardées comme lieux de points, la restriction suivante: et $\xi^{\prime \prime} "$

"Les surfaces $\left[F_{1}\right]$ et $\left[F_{2}\right]$ sont dénuées des singularitis $\vec{j}, \dot{\eta}, \dot{\eta}$

et à lyintroduction du mémoire „Sur les droites multiples des surfaces" la restriction suivante:

"Les équations à la page 4 sant justes si la surface est dénuí des singularites $j, \chi^{\prime}, \eta^{\prime}$ et $\xi$, et les lifuations récipraques si elle $\alpha t$ dérué des singularites $j, \chi, \eta$ et $\xi$. Elles seront donc applicables à la fois à une surface dénuce de toutes ces singularitís.

$\mathrm{Yi}$ à l'un ni à l'autre de ces endroits les restrictions u'altìreront les exposés des résultats; mais il est possible que les singularités dont je parle, si les surfaces en avaient, introduiraient de nouveaux termes dans les équations. Quant aux recherches qui font l'objet pritcipal de mon premrer mémoire, je n'ai pas besoin d'y ajonter aussi une restriction; car les singularités $j$ et $j^{\prime}$ ne s'y présentent que d'unt manière spéciale.

Je ne dis pas que les formules à la page 4 cessent d'etre vraite au delà des limites indiquées ici, mais seulement que wa didaction de ces formules (que je n'ai pas exposée mais où je fais usage, soit des procédés de M. Salmon, soit du principe de correspondance) est alors insuffisante. Pour les singularités $j$ et $j, z$ et $\chi^{\prime} j$ e ne fais que reproduire les formules de M. Cayley (On reciprocal Surfaces. Plil. Trans. 1869, p. 202), et quant aux singularités $\eta$ et $\eta^{1}$ mes résultats sont d'accord avec ceux que ce savant a trouvé par rapport aux "binodes" et aux "biplans"; mais je ne sais pas si M. Cayley veut donner a ses définitions les mêtmes degrés de généralité que j’ai indiqués ici, ou s'il se borne aux cas où les singularités de définition tancrentielle n'ont pas d'autres propriétés ponetuelles que celles qui appartiennent déjà aux singularités ponctuelles quil attribue aux surfaces, tét réciproquement. Cela a lieu pour les surfaces cubiques qui tont un objet principal de ses recherches; mais déjà à l'étude d'one surface de $4^{\text {ur }}$ ordre donée d'une conique double et à laquelle on donne une définition ponctuelle on rencontre des points-jinces qui ont des propriétés tangentielles plus compliquées. Les nombres des singularites de cette surface, tirées des équations dont je viens de parler (Cagley p. 202, Zeuthen p. 4), ne satisfont pas à l'équation que donne M. C'ayley a la page 228. Nous montrerons dans ce qui suit un antre fait qui sera en discordance avec cette derniere équation. (less autres supposcés justes) si lon attribue à la surface des points-clos définis comme je l'ai fait ici. 
En bornant l'étude des surfaces réciproques atix surfaces dénqées des singularités $j, \ddot{j}, \chi, \chi^{\prime}, \eta, \eta^{\prime}, \xi, \xi^{*}$ il sera moins difficile de dé-

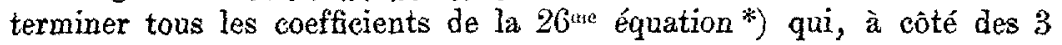
équations (I), des 11 équations (II) - (IV) et (VIII)-(IX) et des 11 équations réeiproques, ont lieu entre les singularités d'une surface. En effet, cette $26^{\text {me }}$ équation sera l'équation (13) [ou (14)] à la page 46 de mon deuxième mémoire.

Je donnerai ici uné autre déduction de la même équation où je ne ferai pas usage de la thêorie de deux surfaces dont les points se correspondent un-à-an, mais seulement du principe de correspondance. Nous appliquerons ce principe à trouver le nombre des plans qui sont à la fois tangents à la-surface et osculateurs à la courbe cuspidale à un mème point de celle-ci. Nous prenons alors pour les points correspondants ( $x$ et $u$ ) dont on cherche les coïncidences les points où le plan tangent et le plan osculateur à un même point de la courbe caspidale rencontrent une droite ixe. A un point $x$ correspondent $\sigma$ points $u$, et, si l'on désigne par $m$ la classe de la déreloppable (d'ordre $r$ enveloppe des plans osculateurs de la courbe cuspidale, à un point $u$ correspondent $m$ points $x$. Il y a done $\sigma+m$. coïncidences diun point $x$ avec un point correspondant $u$. Ces coïncidences ont lieu $1^{0}$ aux $r$ points où la droite fixe rencontre des tangentes de la courbe cuspidale, et $2^{\circ}$ aux points où elle rencontre les plans cherehés. Le nombre de ces plans est donc $\sigma+m-r$.

Une partie des plans trouvés, disons préalablement $\Sigma$, coïncident avec les plans qui sont tangents le long d'une courbe; les points de contact des autres auront la propriété réciproque à celle des plans: celle d'être à la fois points de contact de plans tangents stationnaires avec la surface et points de contact des mêmes plans avec l'arête de rebroussernent de l'enveloppe des plans tangents stationnaires. En déterminant le nombre de ces points d'une manière analogue on trouve l'équation suivante

$$
\sigma+m-r-\Sigma^{\prime}=\sigma^{\prime}+m i-r^{\prime}-\Sigma,
$$

où $m^{\prime}$ est l'ordre de l'arête de rebroússement de l'enveloppe des plans tangents stationnaires, $\Sigma$ le nombre des points qui ont la propriété indiquée et qui se trouvent aux points coniques de la surface. Or on a d'après les relations de Plücker

$$
m=\beta+3 r-3 c, m^{\prime}=\beta^{\prime}+3 r^{\prime}-3 c,
$$

et $\Sigma$ sera égal à la somme des nombres respectifs des droites qui ont

*) Après avoir exposé les autres 25 équations aux pages 3 et 4 je parle à la page 4 (et à la page 46) de deux nouvelles equations (servant à déterminer $\beta$ et $\beta^{\prime \prime}$ ); nais ces équations se réduisent, an moyen des 25 autres, à une seule. (Voir le mémoire de M. Cayley p. 228.) 
aux points coniques $(\mu$-tuples) un contact $(\mu+2)$-ponctuel avec la surface (voir à la page 39 ), ou bien à

$$
\Sigma[\mu(\mu+1)-2 y-3 z]=\Sigma[2 u+\nu] \text {. }
$$

On aura de même i substituer à $\Sigma$ le nombre $\Sigma^{\prime}\left[2 \mu^{*}+v\right]$, On trouve ainsi

$$
\sigma+2 r-3 c+\Sigma(2 \mu+v)=\sigma+2 r^{\prime}-3 e+\Sigma(2 \mu+\nu),
$$

qui est une forme de l'équation cherchée. On peut au moyen des équations à la page 4 la réduire a l'équation (13) à lia page 46.

On pourrait appliquer le même procéde an une surface douée des singularités $j, j$ etc.; mais ne connaissant pas suffisamment les propriêtés de ces singularités, comme je l'ai dit, je ne pourrajs alors déterminer les coefficients. Toutefois cette recherche peut servir it confirmer que lintroduction de ces singularités dans la théorie des surfaces réciproques a été prématurée. Supposons parr exemple que lis surface soit donée de $\chi$ points-clos et de $\chi$ plans-clos. Alors on doit remplacer la formule trouvée paf la suivante:

$$
\begin{aligned}
& \sigma+2 r-3 c+\Sigma(2 \mu+\nu)+A \cdot x \\
= & 5+2 r-3 c+\Sigma(2 \mu+\nu)+A \cdot x,
\end{aligned}
$$

où $A$. est un coefficient inconu mais cutier; mais l'équation de $\mathrm{M}$. Cayley à la page 228 , combinée avec ses autres formules ia la psge 202 , mes formules de la page 4) donnerait a $\chi$ et $\chi$ le coefficient $y_{3} *$ )

* Je saisis loccasion pour répondre a une remarque de M. Notber Nuch. richten der Königl. Gesellachaft der Wisonschatten au Gottingen 1871, p. 27\%): Dan

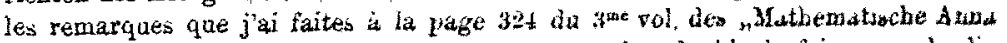
len" sur la représentation d'une surface ara un plan double jo fais, sans le dire je l'avoue, la même supposition que je fass expresoenent dans mon mémoirc sur

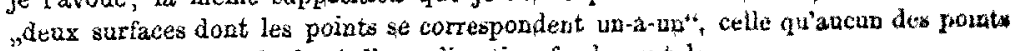
fondamentaux ne soit doue d'une directiou fondamentale. 